patient underwent off-pump stage II palliation at 3 months of age with the placement of a right Glenn shunt, central shunt placement, and ligation of the left-sided superior vena cava above the level of the azygos vein. Postoperative echocardiography showed right ventricular hypertrophy with preserved systolic function, mild neoaortic valve insufficiency, unobstructed PA flow, and mild to moderate obstruction at the junction of the neoaorta and the native descending aorta.

At 4 years of age, the patient underwent pericardial patch augmentation of the neoaorta with excision of the previously fixed ductus arteriosus, anastomosis of the azygos continuity of the inferior vena cava to the left PA (Kawashima procedure), central shunt takedown, and augmentation of the central PA with a $12 \mathrm{~mm}$ GORE-TEX graft (W. L. Gore \& Associates, Newark, Del) on cardiopulmonary bypass. Her postoperative course was uneventful, with echocardiography showing minimal neoaortic valve regurgitation with no obstruction of the neoaorta, PAs, Glenn, and Kawashima anastomoses. Pathologic examination of the excised ductus arteriosus tissue is shown in Figure 2, $C$.

The patient presented at 8 years of age with increasing cyanosis secondary to progressive pulmonary arteriovenous malformations, and thus underwent connection of the hepatic veins to the right PA by using a 16-mm GORE-TEX graft with an uneventful postoperative course.

At most recent follow-up, the patient is 8 years old and growing well. There is excellent right ventricular systolic function, mild tricuspid regurgitation, and no neoaortic valve regurgitation on echocardiography. The neoaorta, cavopulmonary connections, and PAs are all unobstructed. The patient's arterial oxygen saturation is $97 \%$ on room air.

\section{DISCUSSION}

Glutaraldehyde fixation of the ductus arteriosus and off-pump shunt placement may represent an alternative to "hybrid" palliation for higher risk neonates with hypoplastic left heart syndrome physiology. Glutaraldehyde treatment of the ductus arteriosus allowed the deferral of arch reconstruction and circulatory arrest for this patient until her stage III operation at the age of 4 years. The treated ductus was not balloon dilatable or stentable in the catheterization laboratory, which has been a consistent finding in patients treated with this technique. The neoaorta itself remained completely unobstructed from the time of fixation until arch reconstruction was performed. Furthermore, there were no apparent adverse effects from the use of glutaraldehyde for ductal fixation.

An additional advantage of the approach is the avoidance of bilateral PA banding. Separating the pulmonary circulation from the neoaorta facilitates the use of aortopulmonary shunts, which may allow for more predictable pulmonary blood flow.

The limitation of this approach is its complexity. This is a difficult operation that is unlikely to become the standard of care for hypoplastic left heart syndrome. Nevertheless, aspects of this approach may be of use in the treatment of selected patients with single-ventricle congenital heart disease requiring aortic arch augmentation.

\title{
Transcatheter implantation of a newly designed aortic prosthesis in a patient with a mechanical mitral valve
}

\author{
Jan-Philipp Minol, MD, ${ }^{\mathrm{a}}$ Verena Veulemanns, MD, ${ }^{\mathrm{b}}$ Tobias Zeus, MD, ${ }^{\mathrm{b}}$ and Alexander Blehm, MD, ${ }^{\mathrm{a}}$ \\ Düsseldorf, Germany
}

Transcatheter aortic valve implantation (TAVI) in patients with previously implanted mechanical mitral valve prosthesis has been reported to be a challenge, particularly

\footnotetext{
From the Departments of Cardiovascular Surgery ${ }^{\mathrm{a}}$ and Cardiology, Pulmonology, and Angiology, ${ }^{\mathrm{b}}$ University Hospital, Düsseldorf, Germany.

Disclosures: Authors have nothing to disclose with regard to commercial support.

Received for publication May 30, 2014; accepted for publication May 30, 2014; available ahead of print Aug 15, 2014

Address for reprints: Jan-Philipp Minol, MD, Department of Cardiovascular Surgery, University Hospital, Moorenstrasse 5, 40225 Düsseldorf, Germany (E-mail: Jan-Philipp.Minol@med.uni.duesseldorf.de).

J Thorac Cardiovasc Surg 2014;148:e202-4

$0022-5223 / \$ 36.00$

Copyright (c) 2014 by The American Association for Thoracic Surgery

http://dx.doi.org/10.1016/j.jtcvs.2014.05.094
}

with respect to positioning. ${ }^{1-3}$ The new Engager aortic valve prosthesis (Medtronic, Inc, Minneapolis, Minn) is designed to capture the native leaflets of the original aortic valve with its control arms by placing it as close to the native aortic annulus as possible, ${ }^{4}$ potentially perfecting the positioning through tactile feedback. We describe TAVI with an Engager valve in a patient with a previously implanted mitral valve.

\section{CLINICAL SUMMARY}

A 81-year-old white women who had severe symptomatic aortic valve disease with prevailing stenosis (New York Heart Association functional class III) was assigned to undergo aortic valve replacement. Echocardiographic 


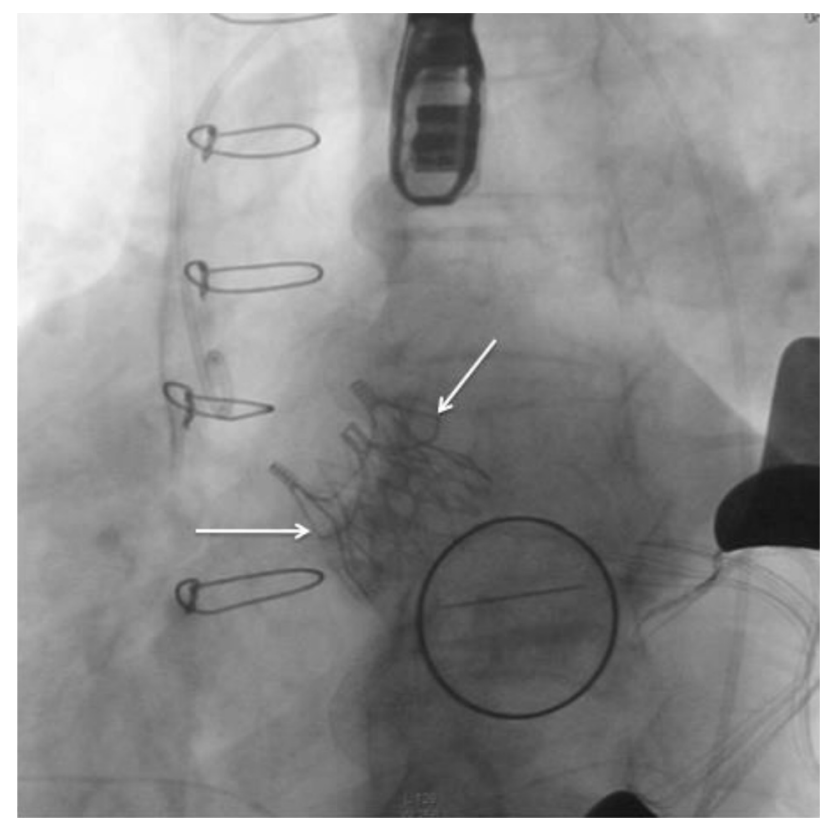

FIGURE 1. The new Engager aortic valve prosthesis is designed to capture the native leaflets of the origin aortic valve with its control arms (arrows) by placing it as close to the native aortic annulus as possible.

examination revealed a planimetric aortic orifice area of 0.6 $\mathrm{cm}^{2}$ and transvalvular gradients of $44 \mathrm{~mm} \mathrm{Hg}$ (peak) and 22 $\mathrm{mm} \mathrm{Hg}$ (mean), with slightly decreased left ventricular ejection fraction $(55 \%)$. The patient's history revealed, in addition to a correction of a tricuspid valve regurgitation by a DeVega annuloplasty, a simultaneous implantation of a 31-mm bileaflet mechanical mitral valve prosthesis ( $\mathrm{St}$ Jude Medical, Saint Paul, Minn) as a result of mitral valve regurgitation 4 years previously. The recurrence of a tricuspid regurgitation was noted, and the mitral prosthesis showed a proper function. The cardiovascular risk profile consisted of type 2 diabetes mellitus, arterial hypertension, and hyperlipidemia. Further comorbidities were atrial fibrillation, pulmonary emphysema, and cardiomegaly. The calculated operative mortality risk according to the logistic euroSCORE and the Society of Thoracic Surgeons score were 20.07 and 5.49, respectively. Considering the elevated perioperative risk, especially as a result of the combination of multimorbidity and reoperative surgery, the heart team decided in favor of a transcatheter procedure.

Transapical TAVI was performed with the patient under general anesthesia in the hybrid operating room. The implantation of a 23-mm Engager aortic valve prosthesis was successfully conducted with the technique described previously. ${ }^{4}$ The newly designed transapical valve system characterized by control arms supported the extremely precise positioning of the aortic valve prosthesis. ${ }^{4}$ It was thus possible to place the prosthesis as close to the aortic valve annulus as possible by capturing the native leaflets for a proper sealing. The tactile feedback during the

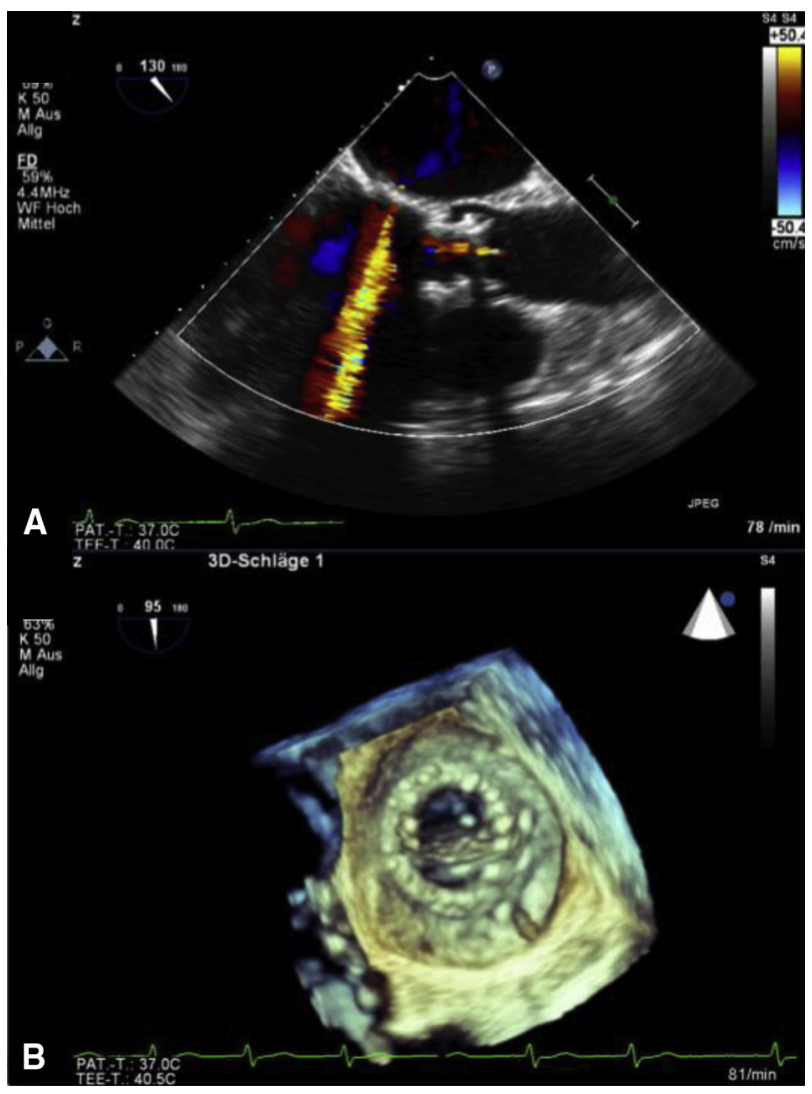

FIGURE 2. A, Despite a very close position of the aortic valve prosthesis to the mitral valve prosthesis, a proper function of both could be found by transesophageal echocardiographic examination. B, The function of the mitral valve prosthesis was not impaired. Both leaflets could move without any interference.

placement avoided the risks of device malpositioning and of interaction with the mitral valve prosthesis (Figure 1).

Perioperative and postoperative transesophageal echocardiography (Figure 2, A) showed a proper function of the aortic valve prosthesis (maximum velocity of shortening of $2.41 \mathrm{~m} / \mathrm{s}$ and maximum pressure change of $26.8 \mathrm{~mm} \mathrm{Hg}$ ) with only a minimal paravalvular leakage. The mechanical mitral valve prosthesis showed correct hemodynamic parameters. The movement of the leaflets was not hindered by the aortic valve prosthesis (Figure 2, B). The postoperative course was uncomplicated. Two months after discharge from hospital, the patient was largely free of symptoms, with New York Heart Association functional class I.

\section{DISCUSSION}

TAVI in patients with previously implanted mechanical mitral valve prosthesis has been reported to be a challenge. ${ }^{1-3}$ A minimum distance between the aortic valve prosthesis and the aortic annulus is required both to avoid any interference with the mitral valve prosthesis, potentially resulting in a sudden device dysfunction, ${ }^{2,3}$ and to allow the 
correct expansion of the aortic valve prosthesis. ${ }^{1,3}$ For this reason, some authors recommend that the implantation be performed as high as possible. ${ }^{2}$ This intention might lead to malpositioning too high in the left ventricular outflow tract, however, potentially causing aortic regurgitation or, even worse, occlusion of the coronary arteries. ${ }^{5}$ It is therefore necessary for the user to find a position that respects these aspects.

The new Engager aortic valve prosthesis is designed to capture the native leaflets of the origin aortic valve with its control arms by placing it as close to the native aortic annulus as possible. ${ }^{4}$ We could prove that the resulting distance ensures not only a correct expansion of the aortic valve prosthesis but also a proper function of a previously implanted mitral valve prosthesis. This perfect positioning with respect to the localization of the two valve prostheses and the coronary arteries can be achieved just by the tactile feedback of the newly designed Engager prosthesis. A previously implanted mitral valve is no longer a challenge for the individual user.

\section{References}

1. Barbanti M, Ussia GP, Latib A, De Marco F, Fiorina C, Santoro G, et al. Transcatheter aortic valve implantation in patients with mitral prosthesis. J Am Coll Cardiol. 2012;60:1841-2

2. Gedikli O, Kutlu M, Civelek A, Ince H. Transcatheter implantation of a CoreValve aortic prosthesis in a patient with a ball-cage mechanical mitral valve. J Heart Valve Dis. 2013;22:697-700.

3. García E, Albarrán A, Heredia-Mantrana J, Guerrero-Pinedo F, Rodríguez J, Hernández-Antolín R, et al. Transcatheter aortic valve implantation in patients with a mechanical mitral valve. Rev Esp Cardiol (Engl Ed). 2011; 64:1052-5.

4. Sündermann S, Holzhey D, Bleiziffer S, Treede H, Falk V. Medtronic Engager ${ }^{\mathrm{TM}}$ bioprosthesis for transapical transcatheter aortic valve implantation. EuroIntervention. 2013;9(Suppl):S97-100

5. Laborde JC, Brecker SJ, Roy D, Jahangiri M. Complications at the time of transcatheter aortic valve implantation. Methodist Debakey Cardiovasc J. 2012; $8: 38-41$

\title{
Redo cardiac surgery in a patient with severe kyphoscoliosis and pectus carinatum: A technical challenge
}

\author{
Syed T. Hussain, MD, ${ }^{a}$ Michelle Capdeville, MD, ${ }^{\mathrm{c}}$ Samir R. Kapadia, MD, ${ }^{\mathrm{b}}$ and Nicholas G. Smedira, MD, ${ }^{\mathrm{a}}$ \\ Cleveland, Ohio
}

Video clip is available online.

Severe chest wall and spinal deformity in patients with Marfan syndrome can impair optimal operative exposure and complicate cardiac surgical operations. We report a case of very high-risk reoperative mitral valve surgery in a patient with severe kyphoscoliosis, pectus carinatum, and severe restrictive lung disease. To the best of our knowledge, successful redo cardiac surgery with such a severe chest wall deformity has not been previously reported.

\section{CLINICAL SUMMARY}

A 24-year-old patient with Marfan syndrome and previous mitral valve replacement with a mechanical valve

From the Departments of Cardiothoracic Surgery ${ }^{a}$ and Cardiovascular Medicine, ${ }^{b}$ Heart and Vascular Institute, and Department of Cardiothoracic Anesthesia, ${ }^{c}$ Anesthesia Institute, Cleveland Clinic, Cleveland, Ohio.

Disclosures: Authors have nothing to disclose with regard to commercial support.

Received for publication June 9, 2014; accepted for publication June 13, 2014; available ahead of print Aug 19, 2014.

Address for reprints: Nicholas G. Smedira, MD, Cleveland Clinic, 9500 Euclid Ave, Desk J4-1, Cleveland, OH 44195 (E-mail: smidern@ccf.org).

J Thorac Cardiovasc Surg 2014;148:e204-6

$0022-5223 / \$ 36.00$

Copyright $(2) 2014$ by The American Association for Thoracic Surgery

http://dx.doi.org/10.1016/j.jtcvs.2014.06.073 performed 10 years earlier presented with severe paravalvular regurgitation. She had severe deformity of the chest wall, with extreme kyphoscoliosis and pectus carinatum (Figures 1 and 2). She also had poor exercise tolerance, and pulmonary function testing showed severe restrictive lung disease (forced vital capacity was $22 \%$ of predicted) with negligible ventilatory reserve, portending a high risk of postoperative respiratory failure. A metabolic stress echocardiogram showed significant pulmonary hypertension with exercise. Computed tomography demonstrated extensive chest wall deformity with right-sided cardiac structures in close proximity to the sternum.

Based on these findings, the patient was deemed a very high operative risk, and an attempt was made at percutaneous device closure of the paravalvular leak (Video 1, A and $B$ ). The devices were successfully placed in the defect from the transseptal approach. While interrogating the devices before release to determine if they interfered with valve function, the devices spontaneously squeezed out of the defect, probably from somewhat unusual twisting motion of the anulus from severe kyphoscoliosis. At this time, the percutaneous closure attempt was aborted without any complications. After extensive discussions, a high-risk operation was offered.

Because of the gross chest deformity and the close proximity of cardiac structures to the sternum (Figure 1), 